\title{
Effect of Side Error on the Static Displacement of Micro-resonator
}

\author{
Fen $\mathrm{GAO}^{1, \text { a }}$, Shuying $\mathrm{HAO}^{2, \mathrm{~b}}$, Chengkun $\mathrm{QI}^{1, \mathrm{c}}$, Jingjing FENG ${ }^{2, \mathrm{~d}}$, Huijie $\mathrm{LI}^{1, \mathrm{e}}$ \\ ${ }^{1}$ School of Maritime, Tianjin University of Technology, Tianjin, 300384, China \\ ${ }^{2}$ Department of Mechanical Engineering, Tianjin University of Technology, Tianjin, 300384, China \\ aemail:yafei0914@163.com, bemail:syhao@ tju.edu.cn, ‘a15266901953a@163.com, \\ demail:jjfeng@ tju.edu.cn, ${ }^{\mathrm{e}}$ tjlglhj@126.com
}

\begin{abstract}
Keywords: Side error of the movable fingers; The initial overlap length; Static displacement; Dc bias voltage
\end{abstract}

\begin{abstract}
In view of the practical situation, sometimes there is side error of the comb capacitor through superposition of the deep reactive ion etching (DRIE) process, and these manufacturing tolerances can lead to the movable comb fingers offset. Using the software of ANSYS to explore the electrostatic-structural coupled field. Then, the effect of sensitive direction offsets on the static displacement of micro-resonator is analyzed, in addition, the different initial finger overlap, dc bias voltage and side error are considered based on the traditional structure, at the same time, the fringe effect and the corner-effect of comb need to be taken into consideration. The ideas and the simulation results in this paper have a guiding significance for improving the performance of the micro-resonators.
\end{abstract}

\section{Introduction}

Micro Electro Mechanical System (MEMS) are systems in which the electronic and mechanical parts are strictly integrated, comb drive structures can be widely seen in electrostatically actuated MEMS devices [1]. Design of comb drive devices requires comprehensive analysis of the MEMS structures. Often in MEMS manufacturing processes, process parameters (such as the gas flow rate, pressure and RF power, etc.) will affect the etching results, which leads the actual etching spacing and design spacing of the comb to be different [2]. Then the difference will change the electrostatic force between comb fingers, and maybe change the static displacement of micro- resonator. However, at present, most of the literature about micro-resonators is based on the ideal structure. Zhao jiangming developed a novel type of micro electrostatic actuator with multi-folded suspensions, which would be able to output large displacement [3]. Huang wei studied that the critical voltage could be in the range of a practical design voltage if there exists a lateral or angular offset based on a two-dimensional model [4]. Zhuyi proved that the side error can cause the instability of the micro-comb structure in horizontal direction [5]. There is no report about the influence of side error on the static displacement of micro-resonator. What's more, the static displacement is one of the most important parameters of the micro-resonators, so it is necessary to discuss the static displacement of resonator on the basis of process error.

\section{The Working Principle of the Static Electric Micro-resonator}

Fig. 1 shows the schematic diagram of electrostatic comb micro-resonators. The movable structure suspended by folded beams at two anchors is actuated in the drive direction by applying a dc bias voltage $V_{d c}$ and two ac driving voltages denoted by $V_{a c}$ and $-V_{a c}$ which have the same amplitude and opposite directions, in addition, its boundary condition are an encastre at beams end. The electric field energy between the stationary and movable fingers changes as the micro-resonator vibrates and the electric field force can be calculated through this change. Small static displacements are generated when a dc bias voltage is applied between the fixed and movable structures. As listed in table 1, the structural parameters and dimensions of electrostatic comb micro-resonators are based on the traditional structure. A Young's modulus of $140 \mathrm{GPa}$, a mass 
density of $2329 \mathrm{~kg} / \mathrm{m}$ and a Poisson's ratio of 0.3 are employed in the simulation, and no material damping has been considered.

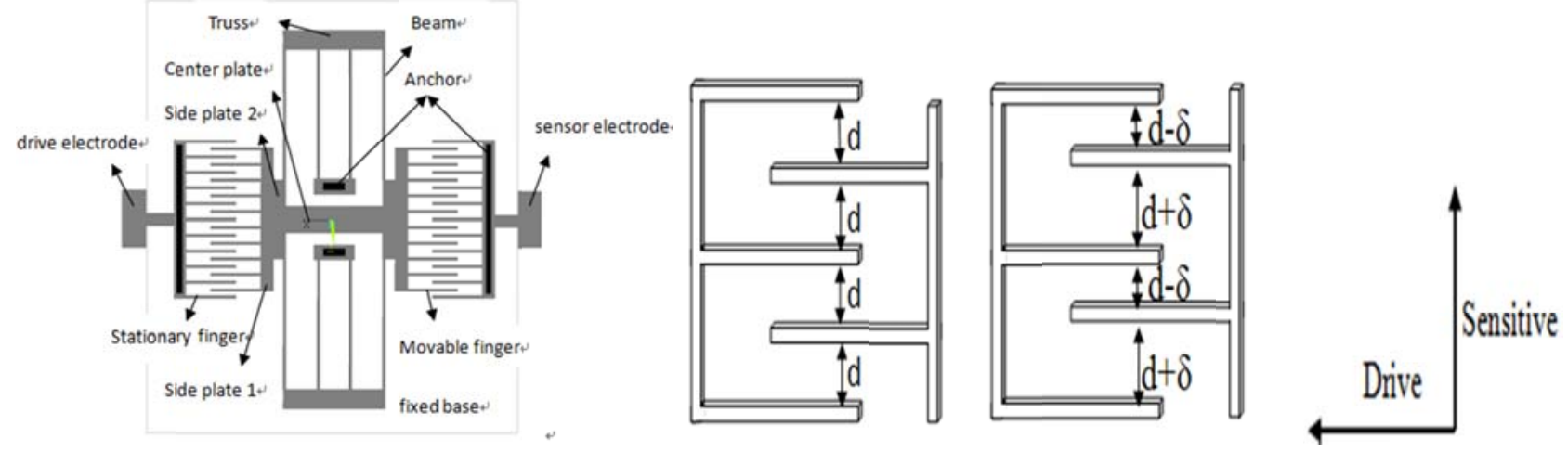

Fig.1 The schematic diagram of

Fig.2 The schematic diagram of comb finger with side error electrostatic comb micro-resonators

Tab.1 Structural parameters and dimensions of electrostatic comb micro-resonators

\begin{tabular}{llll}
\hline Description & $\operatorname{Dims}(\mu \mathrm{m})$ & Description & $\operatorname{Dims}(\mu \mathrm{m})$ \\
\hline Length of comb fingers $(\mathrm{g})$ & 40 & Length of the folded beams & 100 \\
The initial finger overlap $(\mathrm{l})$ & $2-10$ & Width of the folded beams & 2 \\
Width of comb fingers $(\mathrm{w})$ & 2 & Length of the truss & 82 \\
Gap between comb fingers(d) & 4 & Width of the truss & 10 \\
Air gap $\left(\mathrm{h}_{0}\right)$ & 2 & Dimensions of the side plate 1 & $110 \times 10$ \\
Number of comb fingers $(\mathrm{n})$ & 10 & Dimensions of the side plate 2 & $60 \times 10$ \\
Thickness of the structure $(\mathrm{h})$ & 2 & Dimensions of the center plate & $10 \times 39$ \\
\hline
\end{tabular}

The lateral displacement $\mathrm{X}$ of the micro-resonator consists of both static displacement $\mathrm{X}_{0}$ and dynamic displacement $\Delta \mathrm{X}[6]$, the static displacement caused by dc bias voltage $\mathrm{V}_{\mathrm{dc}}$ and the dynamic displacement caused by ac drive voltage $\mathrm{V}_{\mathrm{ac}}$, respectively. The equations of motion can be written as follow:

$$
\mathrm{X}=\mathrm{X}_{0}+\Delta \mathrm{X}
$$

Ideally, the movable finger is set in the center between fixed ones without any offset. In reality, however, due to manufacturing tolerances, there exist sensitive direction offsets, which can significantly affect the "pull-in" voltage [7]. When a dc bias voltage is applied to the device, sensitive forces are generated between adjacent comb fingers. Normally, the sensitive forces balance out. As shown in figure 2, when the movable finger is deviated from the center, the interval from the top to bottom of comb fingers respectively becomes $d-\delta$ and $d+\delta$, which causes the changes of the electrostatic force both in drive direction and sensitive direction. In general, the side error of movable comb fingers is small. According to the range of the microstructure process's allowable tolerances [8], when the gap between comb fingers is $4 \mu \mathrm{m}$, the maximum side error of movable fingers is $0.4 \mu \mathrm{m}$.

\section{The Analysis of Micro-resonator's Static Displacement}

According to the design parameters' range of the micro-resonator, the range of the side error is from $0 \mu \mathrm{m}$ to $0.4 \mu \mathrm{m}$, the range of dc bias voltage is from $20 \mathrm{v}$ to $100 \mathrm{v}$ and the initial finger overlap is controlled from $2 \mu \mathrm{m}$ to $10 \mu \mathrm{m}$. Hence, under these conditions, the static displacement of micro-resonator will be analyzed.

As shown in figure 3, the entity model of the electrostatic and regional structure was established and the air model around the comb fingers was established to simulate the electrostatic field. Then, the structure and electrostatic area were meshed. Firstly, assign the air model area the solid122 element to create static physical environment, then define the material properties and the excitation voltage. After that, set the element type of the structure as zero element type, and write the electrostatic physical environment to the physical files. Secondly, establish the structural physical 
environment instead of the electrostatic physical environment, chose solid95 element, meanwhile define the material properties, the entity model's boundary conditions and loads. Then, set the element type of electrostatic as zero element type, chose equation solvers and options. After that, write the structural physical environment to the physical files. Finally perform ESSOLV macros.

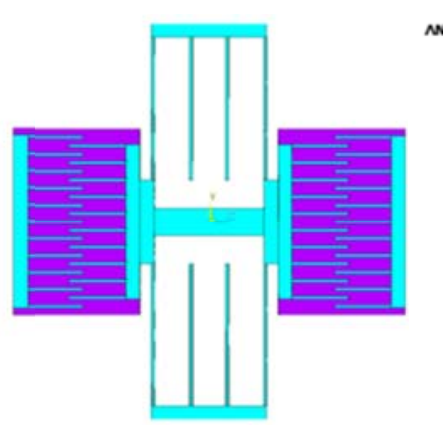

Fig.3 Finite element model of micro-resonators

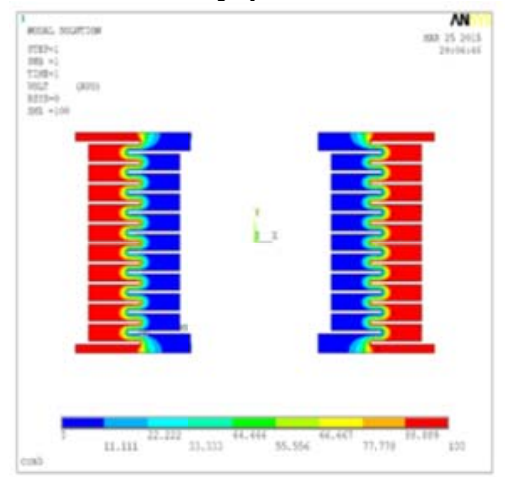

Fig.4 Fringe effect of electrostatic micro-resonators

There are some differences between the actual edge electric field distribution and the expected edge electric field distribution, as can be clearly seen from the figure 4 . Because the gap between the comb fingers is roughly equal to the thickness of the comb fingers, comb electrostatic field can't completely be regarded as parallel plate capacitor which neglect the fringe effect and the cornereffect, the fringe effect and the corner-effect of comb should be fully considered, then the drive force and static displacement will be influenced by the fringe effect and the corner-effect.

\section{Effect of Side Error on the Static Displacement of Micro-resonator}

Based on the above analysis, the effect of the initial finger overlap and the dc bias voltage and the side error on the static displacement is discussed, the followings have been achieved in this paper, and the result is helpful to design micro-resonator to some extent.

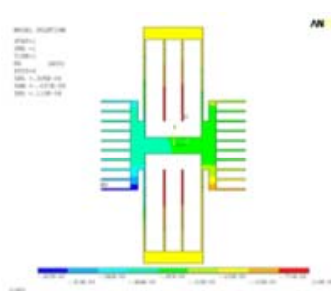

(1) $\mathrm{V}_{\mathrm{dc}}=20 \mathrm{v}$

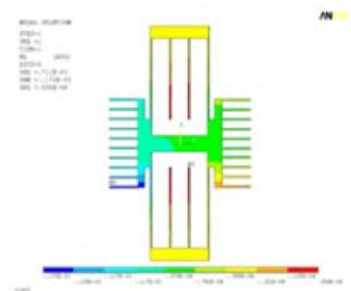

(2) $\mathrm{V}_{\mathrm{dc}}=100 \mathrm{v}$

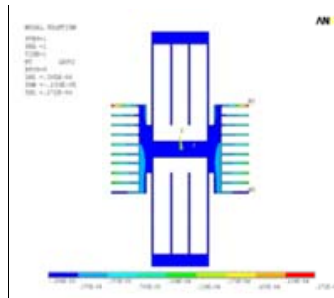

(1) $\mathrm{V}_{\mathrm{dc}}=20 \mathrm{v}$

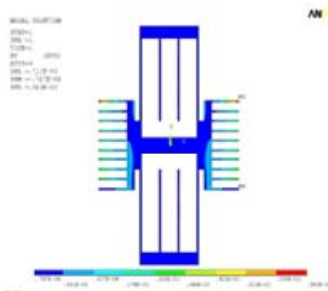

(2) $\mathrm{V}_{\mathrm{dc}}=100 \mathrm{v}$

Fig. $5 \delta=0 \mu \mathrm{m}, 1=2 \mu \mathrm{m}$, the static displacement $\quad$ Fig. $6 \delta=0 \mu \mathrm{m}, 1=2 \mu \mathrm{m}$, the static displacement of drive direction

of sensitive direction

As shown in figure 5 and figure 6, when the movable finger is in the center of the gap between comb fingers, the static displacement of micro-resonator is almost zero. The electrostatic force is balanced by the spring force at equilibrium. In fact the force of every finger has tiny difference due to the fringe effect and the corner- effect, so that the static displacement of each finger is slightly different. Hence, as both the initial finger overlap and the dc bias voltage are big or small, the comb fingers are approximately parallel.

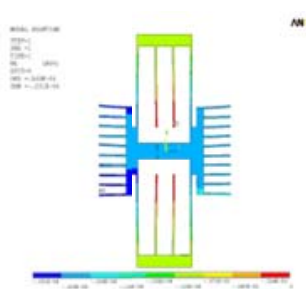

(1) $\mathrm{V}_{\mathrm{dc}}=20 \mathrm{v}$

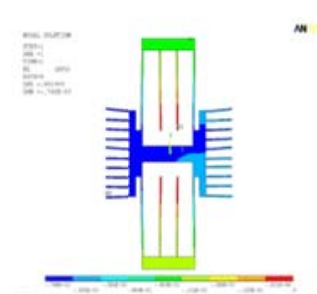

(2) $V_{d c}=100 \mathrm{v}$

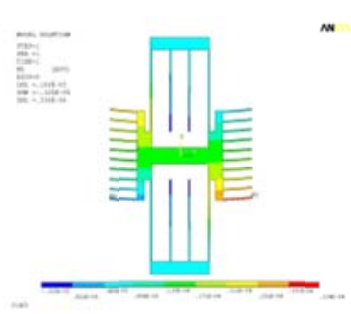

(1) $\mathrm{V}_{\mathrm{dc}}=20 \mathrm{v}$

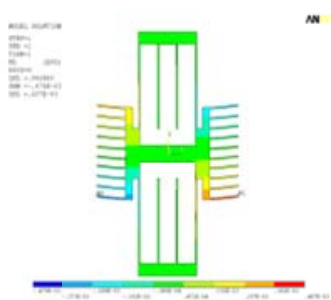

(2) $\mathrm{V}_{\mathrm{dc}}=100 \mathrm{v}$

Fig. $7 \delta=0.2 \mu \mathrm{m}, 1=2 \mu \mathrm{m}$, the static displacement Fig. $8 \delta=0.4 \mu \mathrm{m}, 1=10 \mu \mathrm{m}$, the static displacement of drive direction

of drive direction 
As shown in figure 7 or figure 8 , when the side error of movable fingers exists, the micro-resonator has static displacements along the drive direction, the lateral forces are generated between adjacent comb fingers, and the folded beams become deformed. There is the maximum displacement of the comb fingers and plates, but the displacement of the folded beam and truss is relatively small; Contrast in figure 7 (1) (2) or figure 8 (1) (2), while the initial finger overlap is the same, the larger the dc bias voltage, the bigger the static displacement along the drive direction.

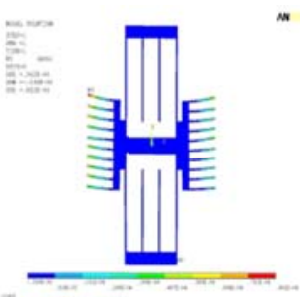

(1) $\mathrm{V}_{\mathrm{dc}}=20 \mathrm{v}$

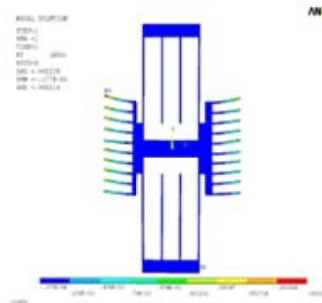

(2) $\mathrm{V}_{\mathrm{dc}}=100 \mathrm{v}$

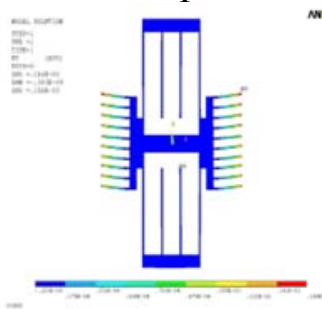

(1) $\mathrm{V}_{\mathrm{dc}}=20 \mathrm{v}$

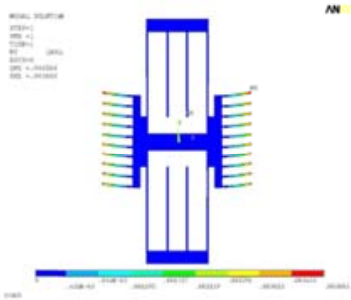

(2) $\mathrm{V}_{\mathrm{dc}}=100 \mathrm{v}$

Fig. $9 \delta=0.4 \mu \mathrm{m}, 1=2$, the static displacement Fig. $10 \delta=0.4 \mu \mathrm{m}, 1=10 \mu \mathrm{m}$, the static displacement of sensitive direction of sensitive direction

As shown in figure 9 or figure 10, when the side error of movable fingers exists, there is the maximum displacement along the sensitive direction at the end of comb fingers, and the comb fingers present the upward trend. The displacement depends on both the initial finger overlap and the applied voltage. Contrast in figure 9 (1) (2) or figure 10 (1) (2), while the initial finger overlap is the same, the larger the dc bias voltage, the bigger the static displacement of sensitive direction.

Compare figure 8 with figure 11 , as both the initial finger overlap and the dc bias voltage are the same, but the side error is different; the static displacement of drive direction is slightly different. Compare figure 10 with figure 12, as both the initial finger overlap and the dc bias voltage are the same, the larger the side error, the bigger the static displacement of sensitive direction. Compare figure 7 with figure 11, not only the side error of movable fingers but also the dc bias voltage is the same, but the initial finger overlap is different, the static displacement of drive direction is slightly different. Compare figure 9 with figure 10, as both the side error and the dlc bias voltage are the same, the larger the initial finger overlap, the bigger the static displacement of sensitive direction.

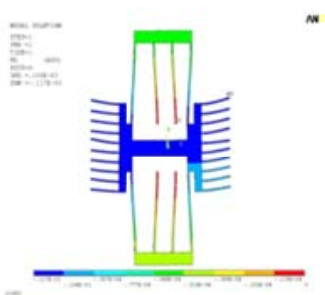

(1) $\mathrm{V}_{\mathrm{dc}}=20 \mathrm{v}$

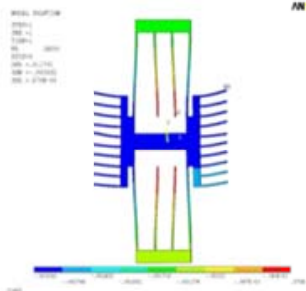

(2) $\mathrm{V}_{\mathrm{dc}}=100 \mathrm{v}$

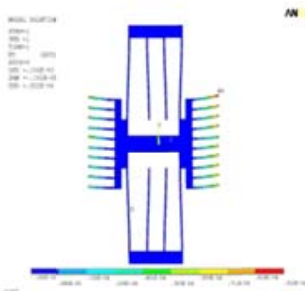

(1) $\mathrm{V}_{\mathrm{dc}}=20 \mathrm{v}$

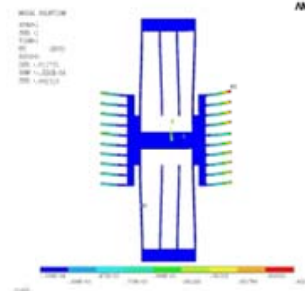

(2) $\mathrm{V}_{\mathrm{dc}}=100 \mathrm{v}$

Fig. $11 \delta=0.2 \mu \mathrm{m}, \mathrm{l}=10 \mu \mathrm{m}$, the static displacement Fig. $12 \delta=0.2 \mu \mathrm{m}, \mathrm{l}=10 \mu \mathrm{m}$, the static displacement of drive direction of sensitive direction

\section{Conclusion}

This paper demonstrates that when the movable finger is in the center of the gap between comb fingers, both the initial finger and the dc bias voltage have no effect on the static displacement. However, when a small side error exists, both the initial finger and the dc bias voltage have effect on the static displacement. While both the initial finger overlap and the side error are the same, the larger the dc bias voltage, the bigger the static displacement along both the drive direction and the sensitive direction. As both the initial finger overlap and the dc bias voltage are the same, the larger the side error, the bigger the static displacement of sensitive direction, but the different side error has no effect on the static displacement of drive direction. As both the side error and the dc bias voltage are the same, the larger the initial finger overlap, the bigger the static displacement of sensitive direction, but the different initial finger overlap have no effect on the static displacement of drive direction. 


\section{Acknowledgements}

This work was financially supported by the National Natural Science Foundation (11372210), the Doctoral Scientific Fund Project (20120032110010) and the research plan of undergraduate teaching quality and teaching reform in Tianjin City (C03-0802).

\section{Reference}

[1] Thielicke E, Obermeier E. Micro actuators and their technologies. Mechatronics. 2000, 10: 431-455.

[2] QiangDai, QiYu, PingShu. Capacitance and Electrostatic Force Distribution Models of Micro Sensors Based on Comb Finger Gaps' MEMS Process Error. Instrument Technique and Sensor. 2010.

[3] Jiang-ming Zhao. Mechanical Performance Analysis and Experiment Research on Laterally Driven Electrostatic Comb Silicon Micro Resonators [D]. Shanghai university, 2006.

[4] Wei Huang, Ganyu Lu. Analysis of lateral instability of in-plane comb drive MEMS actuators based on a two-dimensional model. Sensors and Actuators, 2004.

[5] YiZhu, DanGuo. Couple Simulative Analysis for Drive Characteristic of Micro-comb Structure. Engineering Mechanics, 2008.

[6] Yan-bin Li. Analysis and Optimization Design of Micro-Electromechanical Systems with Electrostatic Comb [D]. Huazhong University of science and technology, 2008.

[7] JieWu. Investigation of Micro cantilever Electromechanical System Dynamic [D]. Shanghai Jiao Tong University, 2012.

[8] Li-yun Zheng, You-ping Gong, Yan-hui Liu. Based on the ANSYS electrostatic micro actuators and phenomenon analysis [J]. China's New Technology and Products, 2014, 29 and 30 . 